\title{
Variatio delectat! - Ist die Verwendung idiomatischer Ausdrücke unkreativ?
}

\section{Bemerkungen zur Hassliebe sprachlicher „Konserven“}

\author{
- [...] Allez, dites-moi, où est passé le livret? \\ - Quel livret?
}

Sincèrement, il ne lui revenait en tête aucune histoire de livret.

- Ne jouez pas les vierges effarouchées, Montalbano!

Ce furent précisément ces mots, «ne jouez pas les vierges effarouchées », qui le déchaînèrent. Il détestait les expressions toutes faites, les façons de parler, ça lui faisait venir les nerfs d'une manière irrépressible.

Cette fois, ce fut lui qui donna un grand coup de poing sur le bureau, lequel réagit en faisant crac crac.

- Mais de quelle connerie de livret vous déparlez?

- Hé ! Hé ! ricana le Questeur. On se sent pas le nez propre, Montalbano ?

Il devina qu'après les vierges effarouchées et le nez propre, allait arriver une autre expression de ce type et qu'il prendrait Bonetti-Alderighi au collet et le ferait mourir étouffé. Miraculeusement, il a réussi à ne pas réagir, à ne pas ouvrir la bouche.

(Camilleri 2003: 39) ${ }^{1}$

1 Das Zitat wurde absolut originalgetreu wiedergegeben! Es wurde im Übrigen vorgezogen, die französische Übersetzung aus dem Italienischen zu zitieren, die wesentlich besser gelungen zu sein scheint als die deutsche:

„Und jetzt reden Sie: Wo steckt das Sparbuch?“

„Welches Sparbuch?“

Er konnte sich wirklich an kein Sparbuch erinnern.

„Spielen Sie doch nicht den Ahnungslosen, Montalbano!“

Bei diesen Worten, „spielen Sie doch nicht den Ahnungslosen“, rastete der Commissario aus. Er hasste Plattitüden und sprachliche Klischees, sie brachten ihn zur Weißglut. Diesmal schug er mit der Hand auf den Tisch, dass es krack, krack machte.

„Von welchem Sparbuch faseln Sie da?“

„Oho!“ höhnte der Questore. „Wir haben wohl was auf dem Kerbholz, was, Montalbano?“

Er wusste, wenn auf den Ahnungslosen und das Kerbholz noch ein Satz von dieser Sorte folgte, würde er Bonetti Alderighi am Hals packen und zudrücken, bis er erstickte. Wie durch ein Wunder schaffte er es, nicht zu reagieren, keinen Ton zu sagen. (Camilleri 2003: 43f.) 
Wie Andrea Camilleris wunderbarer Commissario Montalbano haben wir im Grunde alle sprachliche Vorlieben oder Abneigungen, was die Verwendung bestimmter Sprechweisen, Ausdrücke oder Ausdruckstypen angeht. Häufig werden dabei gerade vorgefertigte Ausdrücke inkriminiert, die dem Adressaten nicht nur deshalb nicht gefallen, weil sie vorgefertigt sind, also eine gewisse Stabilität und Verwendungshäufigkeit ${ }^{2}$ aufweisen, sondern vor allem deshalb, weil sie Bilder enthalten, die dem Hörer aus unterschiedlichen Gründen missfallen. Camilleri, Montalbanos geistiger Vater, verwendet ja selbst in diesem kurzen Ausschnitt eine ganze Reihe sogar metaphorischer Phraseme. Es kann also nicht generell um die Ablehnung vorgeformter Ausdrücke gehen, die in der Kommunikation schlicht unverzichtbar sind. Routineformeln (Prost! Guten Morgen! Herzlichen Glückwunsch!), Kollokationen (sich die Zähne putzen, ins Gerede kommen, die Kontrolle verlieren) oder selbst bestimmte Teilidiome (blinder Passagier, fließend Deutsch sprechen, nicht im Entferntesten) sind zum Ausdruck bestimmter Sachverhalte schlicht unverzichtbar. Was im Übrigen auch für Metaphern gilt, die in der Sprache laut Lakoff/Johnson (1998) allgegenwärtig sind. Um derartige Phraseme oder Metaphern kann es folglich nicht gehen, wenn „sprachliche Konserven“ abgelehnt und kritisiert werden, da sie zum Ausdruck bestimmter Sachverhalte unerlässlich sind.

Wray/Perkins (2000) weisen überdies darauf hin, dass eine Balance zwischen holistischer und kreativer Verbalisierung nicht nur normal, sondern notwendig ist:

[...], our view is that the best deal in communicative language processing is achieved by the establishment of a suitable balance between creative and holistic processes. The advantage of the creative system is the freedom to produce or decode the unexpected. The advantage of the holistic system is economy of effort when dealing with the expected. [...] Without the rule-based system, language would be limited in repertoire, clichéd, and, whilst suitable for certain types of interaction, lacking imagination and novelty. In contrast, with only a rulebased system, language would sound pedantic, unidiomatic and pedestrian. It would require full access to all of the language faculties at all times, and there would be no 'short cuts'. (Wray/Perkins 2000: 11)

Im vorliegenden Beitrag wird es im Übrigen nicht um Phraseologismen im Allgemeinen gehen, sondern vielmehr um solche, die bildhaft sind, also satzwertige

Insbesondere würde kaum jemand am Teil-Idiom den Ahnungslosen spielen Anstoß nehmen. Besser wäre hier meines Erachtens ein Idiom wie spielen Sie doch nicht die Unschluld vom Lande gewesen.

2 Beide Begriffe, sowohl Stabilität als auch Verwendungshäufigkeit, sind zentrale Elemente gängiger Definitionen phraseologischer Ausdrücke. 
oder nicht-satzwertige, metaphorische oder nicht-metaphorische idiomatische Ausdrücke. ${ }^{3}$ Zunächst wird die Kritik der praktischen Stilistik an „sprachlichen Konserven“ Erkenntnissen zur starken Frequenz formelhafter Wendungen in Texten gegenüber gestellt (Punkt 2). Angesichts unbefriedigender Abgrenzungskriterien dessen, was die Stilistik als „Konserve“ betrachtet, wird dann beleuchtet, was „folk“ für sprachlich unkreativ oder abgenutzt hält (Punkt 3). Im Gegensatz dazu wird dann im 4. Punkt die Position der linguistischen Sprachkritik skizziert und im Anschluss an Gülich (2008) dargestellt, dass vorgeformte Ausdrücke als Formulierungsressource dienen. Abschließend soll überlegt werden, ob es vorstellbar ist, in einem literarischen Text vorhandene Idiome durch nicht-phraseologische semantische Äquivalente zu ersetzen.

\section{Zum Gebrauch vorgeformter Ausdrücke - Stilististische Desiderata vs. Gebrauchs- frequenz}

Die übermäßige Verwendung dieser abgeschmackten Verbindungen ist eines der offensichtlichsten Zeichen fehlender Originalität. Je ausgeprägter das Bemühen um guten Stil ist, deso seltener trifft man auf derartige Wendungen, die jeder schon einmal gehört oder gelesen hat, während es davon in der schlechten Literatur nur so wimmelt, Zeitungstexte voll davon sind. Dem wahrhaftigen Literaten hingegen ist daran gelegen, immer neue Ausdrücke zu schaffen, so wie er auch die Verwendung von Bildern variiert (Bally 1909: 74; meine Übersetzung). ${ }^{4}$

Laut Stein (1995) erhebt sich die praktische Stilistik, zweifellos von Bally (1909; siehe vorstehendes Zitat) beeinflusst, unter Verabsolutierung des Prinzips Variatio delectat! ${ }^{5}$ generell gegen jegliche Wiederholung und insbesondere die von vorgeformten Ausdrücken, die als „Konserven“ bezeichnet werden:

3 Auf die von Burger (1989) getroffene Unterscheidung von bildhaft, bildlich, bildstark wird hier verzichtet.

4 Hier das französische Original: „[...], l'emploi abusif de ces groupements banals est un des signes les plus évidents de l'absence d'originalité. Plus l'empreinte du style est forte, moins on y surprend de ces tours que tout le monde a lus ou entendus, tandis qu'ils pullulent dans littérature de bas étage ; la prose des journaux en est farcie. Le véritable littérateur tend à renouveler les séries, comme il renouvelle les images.“ (Bally 1909: 74)

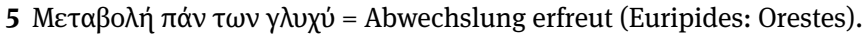


Die praktische Stilistik lebt von 'Feindbildern', die als 'Stilkrankheiten' apostrophiert werden: Behörden-/Amts-/Verwaltungs-/Kanzlei- oder Papierdeutsch, [...] und nicht zuletzt 'Formeldeutsch' sind längst nicht alle, aber die meistbekämpften Sprachschludereien. Für unseren Zusammenhang von Bedeutung ist das der Ausdruckswiederholung angehängte Stigma: Gemäß der rhetorischen Maxime 'Variatio delectat!' gilt der wiederholte Gebrauch desselben Wortes, desselben Ausdrucks, derselben Wendung in einem Text als verpönt. Im gleichen Atemzug, in dem die Abneigung gegen Wiederholung ausgesprochen wird, wird unisono gegen Redensarten, Modewörter und (leere) Phrasen gewettert, allzu undifferenziert bisweilen. Bei der Lektüre von Stilratgebern drängt sich unweigerlich der Eindruck auf, als setzten die Stillehrer Phraseologie gleich mit Phrasendrescherei und Phrasenhaftigkeit. (Stein 1995: 89f.)

Davon abgesehen, dass 'Formelhaftigkeit' in keiner Weise definiert oder differenziert wird, bleiben auch die äußerst zahlreichen Formen der repetitio der literarischen Stilistik völlig unberücksichtigt. ${ }^{6}$ Offensichtlich sind Wiederholungen dem Literaten vorbehalten, der normal Sterbliche hat darauf zu verzichten. Dieser kategorischen Ablehnung „sprachlicher Konserven“, die aufgrund ihrer Wiederholung von bereits Existierendem als unkreativ gelten, stehen in zunehmendem Maße linguistische Arbeiten gegenüber, die auf die starke Frequenz formelhafter Ausdrücke („formulaic expressions“) in unterschiedlichen Texttypen schließen. So meint Altenberg (1998):

Corpus research [...] has made a number of estimates of the proportion of formulaic material in normal language, stretching as high as 80 per cent. (zitiert nach Wray 2000: 466)

Wray/Perkins (2000) sind ein wenig vorsichtiger, wenn sie von „nur“ $70 \%$ ausgehen:

If we take formulaicity to encompass, as some do, also the enormous set of 'simple' lexical collocations, [...], then possibly as much as $70 \%$ of our adult native language may be formulaic [...]. A range of corpus studies [...] have shown that the patterning of words and phrases in ordinary language manifests far less variability than could be predicted on the basis of grammar and lexicon alone, and in fact most natural language, written or spoken, appears to consist largely of collocational 'sets' or 'frameworks' [...]. (ebd.: 1f.)

Um zwei weitere Untersuchungen zu nennen: Erman/Warren (2000) ermitteln die Vorgeformtheit von 52\% schriftlichen und 58\% mündlichen Sprachproduktionen, van Lancker-Sidtis/Rallon (2004) erheben, dass 25\% der „Sätze“ des Skripts des

6 Beispielsweise Anadiplose, Anapher, Diaphora, Epanadiplose, usw. - Siehe zur Frage der Wiederholung aus unterschiedlichen Perspektiven auch „Répétitions et genres“ (Magri-Mourgues/ Rabatel (Hgg., 2015a) oder „Pragmatique de la répétition“ (2015b). 
Films „Some like it hot“ aus „,speech formulas, idioms, proverbs and other formulaic expressions“ bestehen.

Nun sagt diese Tatsache an sich nichts über die Kreativität von „formulaic speech“ aus, denn Sprecher könnten einfach davon abstrahieren. Andererseits könnten die zitierten Beobachtungen jedoch darauf hinweisen, dass es schlicht unmöglich ist, ohne den Rückgriff auf Vorgeformtes zu verbalisieren. Was dann im Umkehrschluss beweisen würde, dass nicht alles, was 'formulaic' ist, gleichzeitig auch unkreativ sein kann, da es eben einfach normal ist. Man kritisiert ja auch keine Routineformeln, die immer wieder bemüht werden (müssen).

Vergessen werden soll auch nicht, dass bereits seit geraumer Zeit Erkenntnisse bzgl. der Präformierung von Sprache vorliegen. So erwähnt Michel Bréal schon 1872 ,locutions toutes faites“, und Hermann Paul behauptet nicht später als 1970 [1880]: „Erst wo Sprechen und Verstehen auf Reproduktion besteht, ist Sprache da.“ Eine Feststellung, die im völligen Gegensatz zur Ablehnung alles Vorgeformten der praktischen Stilistik steht. Deren Haltung wird im Übrigen auch von der häufig im Anschluss an Bally (1909) vertretenen Hypothese in Frage gestellt, die darauf abhebt, dass idiomatisch-bildhafte Ausdrücke prinzipiell expressiver als deren nicht-phraseologischen Äquivalente seien. ${ }^{7}$ So beispielsweise Riesel (1970: 25): ,Je höher die Bildkraft der Sprechweise in Lexik und Phraseologie ist, desto stärker wird ihr Gefühlswert“.

Bardosi/Ettinger/Stölting (2003: X) sind davon überzeugt, dass der bildhafte Charakter der Redewendung „tirer les vers du nez de $\mathrm{qn}^{\text {“8 }}$ gegenüber seinem nichtphraseologischen Pendant ,questionner habilement qn pour lui faire dire des choses qu'il veut cacher“ einen „semantischen Mehrwert“ (ebd.) besitzt, der in einer emotional-expressiven Färbung besteht. Gréciano (1983: 235) geht ebenfalls davon aus, dass im Verlaufe der textuellen Entwicklung eine idiomatische Paraphrase eines nicht-idiomatischen Ausdrucks hin zu mehr Expressivität tendiert, während die nicht-phraseologische Paraphrase eines idiomatischen Ausdrucks mehr Rationalität oder Sachlichkeit impliziert als dieser.

Auch wenn Schmale (2010) im Anschluss an Drescher (1997) nachweist, dass es sich dabei allenfalls um ein Expressivitätspotenzial handelt, das sich in bestimmten Interaktionskonstellationen entfalten kann, aber keineswegs muss, steht die Expressivitätshypothese diametral der Behauptung der praktischen Stilistik gegenüber. Die Verwendung eines bildhaften Ausdrucks kann nicht gleich-

$7 \mathrm{Zu}$ theoretischen Implikationen des Expressivitätsbegriffs siehe Monneret (2010).

8 Deutsch: jmdm. die Würmer aus der Nase ziehen und jmdn. so geschickt befragen, dass er Dinge preisgibt, die er eigentlich verbergen wollte. 
zeitig unkreativ, phrasenhaft, banal, dann aber wieder besonders ausdrucksstark oder expressiv sein.

Angesichts der Tatsache, dass fehlende Kreativität offensichtlich allein über die Tatsache der Wiederverwendung bereits Existierenden, also Vorgeformten, begründet wird, soll im folgenden Abschnitt überlegt werden, ob laienlinguistische Einstellungen von „folk“, d.h. von „normalen“ Mitgliedern der Sprachgemeinschaft, Aufschluss bzgl. der Kriterien erbringen können, die Sprecher anlegen, wenn sie einen Ausdruck als nicht kreativ bewerten. ${ }^{9}$

\section{Laienlinguistische Sprachkritik}

Stein (2016) zufolge zeichnet sich laienlinguistische Sprachkritik dadurch aus, dass Nicht-Linguisten, meist ohne theoretisch-methodische und vor allem empirische Grundlage, ihr Missfallen an bestimmten Gebrauchsformen im Hinblick auf nicht definierte Sprachnormen äußern. (vgl. ebd.: 139) ${ }^{10}$

Zu ergänzen wäre, [...], dass laienlinguistische Sprach- und Stilkritik durch die Bereitschaft zu ironisierender , u.U. polemisierender Darstellung, kurs: zur auffälligen Bewertung des öffentlichen Sprachgebrauchs geprägt ist. (Stein 2016: 139)

Negativ schlagen Kritikpunkte auf unterschiedlichen Ebenen zu Buche: Sie erstrecken sich auf die Sprache selbst bzw. die ästhetische Dimension des Sprachgebrauchs („Sprachverunglimpfung“), auf die Gebrauchsfrequenz und die damit zusammenhängenden Abnutzungseffekte (gehäufter bzw. „unreflektierter Gebrauch“, Abgedroschenheit und Abgegriffenheit) sowie - mit Abstand dominant - auf die ausgedrückten Sachverhalte (Inhaltsarmut bzw. Inhaltslosigkeit und „Sinnentleertheit“ bzw. „Hohlheit“) und die dahinter stehenden verantwortlichen Akteure („Hohlköpfe“). Weit verbreitet bei Laien-Sprachkritikern ist also das Argumentationsmuster, ausgehend von einer bestimmten Art des Sprachgebrauchs Rückschlüsse zu ziehen auf die Sprachverwender und diese nicht nur zu diffamieren, sondern ihnen unlautere Motive und Täuschungsabsicht zu unterstellen (Verschleierung und Verdecken bzw. Ersetzen der Realität bzw. der Wahrheit). (ebd.: 143) ${ }^{11}$

Die Phänomene, aufgrund derer „folk“, Laien, „normale“ Sprachbenutzer, die über keine linguistische Ausbildung verfügen, bestimmte vorgeformte Konstruktionen brandmarken, sollen im Folgenden anhand von Beispielen aus einer Kurz-

9 Siehe Schmale (2008) zu einer Diskussion des „folk“-Begriffes.

10 Die Nähe laienlinguistischer Kritik zu der von der praktischen Stilistik geübten (siehe Punkt 2) ist im Übrigen unübersehbar, kann hier aber nicht diskutiert werden.

11 Für eine Reihe von Literaturhinweisen auf laienlinguistische, teils bissige Kritik an formelhafter Sprache siehe Stumpf (2016). 
umfrage zur Expressivität (Schmale 2010) illustriert werden. So gaben Befragte zur Begründung der Tatsache, dass sie bspw. „sehr gerne essen“ für expressiver halten als „kein Kostverächter sein“, als Argumente u.a. an, dass das Idiom gegenüber dem erstgenannten nicht-phraseologischen Ausdruck altmodisch oder veraltet sei (Schmale 2010: 112). Woraus man schließen kann, dass die (Wieder-) Verwendung ${ }^{12}$ depräferiert wird, weil sie auf veraltetes Sprachmaterial zurückgreift, also nicht den stilistischen Anforderungen modernen Sprachgebrauchs entspricht und somit als unkreativ gilt.

Beim allsonntäglich stattfindenden Fußballstammtisch des Privatsenders Sport 1 gibt es ein „Phrasenschwein“, in das Teilnehmer der Gesprächsrunde immer dann drei Euro einwerfen müssen, wenn der Moderator entscheidet, sie hätten eine Phrase produziert. Als „Phrase“ sanktioniert werden hier idiomatische Wendungen wie neue Besen kehren gut (bei Trainerwechsel), Truismen wie ein Spiel dauert 90 Minuten (wenn noch in der allerletzten Minute ein Tor geschossen wird), aber auch einfache Kollokationen wie Fehler passiern (eben), wobei letztere vom Moderator sogar als „Superphrase“ tituliert wird. Kriterien für das, was als „Phrase“ sanktioniert wird, werden nie angegeben, viele Phraseme, auch idiomatisch-bildhafte Ausdrücke, bleiben ungesühnt. Es scheint, dass der Obolus - allerdings völlig unsystematisch - dann fällig wird, wenn ein Ausdruck stereotyp für einen im Fußball stereotypen Sachverhalt (wieder)verwendet, dabei aber wahrscheinlich als zu häufig für die betreffende Situation gebraucht wird, damit als abgenutzt und folglich auch unkreativ gilt. Man könnte sich fragen, ob beim Sprechen über Situationen, die durch immer wiederkehrende Elemente charakterisiert werden, nicht auf Routineformeln vergleichbare formelhafte Ausdrücke zurückgegriffen werden muss, da das sprachliche Material für die Bezeichnung der entsprechenden Situationen eben begrenzt ist. ${ }^{13}$

Diese Beobachtung könnte auch auf Ausdrücke zutreffen, die in bestimmten Situationen zur Charakterisierung bestimmter Tatbestände fast systematisch bzw. gar routinehaft wieder auftauchen, z.B. prendre son mal en patience oder prendre les usagers en otage ${ }^{14}$ (bei Streiks in Frankreich). Im (Neu-)Deutschen könnte eine negative Einstellung formelhafte Ausdrücke wie das geht gar nicht oder Wie + ADJ + ist das denn? (vgl. Auer 2016) betreffen, die aufgrund ihres stereotypen Charakters

12 Probanden bezeichnen das Idiom auch als „bekannte Floskel“.

13 So wie auch Lexeme zur Bezeichnung von Objekten oder Sachverhalten per Konvention erstarrt sind.

14 Für das erste Phrasem existiert ein deutsches Äquivalent, nämlich „sich in Geduld fassen“; für das zweite allerdings nicht. Wörtlich übersetzt bedeutet es: „die Benutzer als Geiseln nehmen“. 
und ihrer Undifferenziertheit missfallen. Aber eben nicht prinzipiell allen Mitgliedern der Sprachgemeinschaft, sondern in diesem Fall dem Autor des vorliegenden Beitrags einfach aufgrund persönlicher Präferenzen.

Wenn auch ein einzeln auftretendes Idiom akzeptabel sein mag, wird die Häufung besonders bildhafter Phraseme als unelegant, schwerfällig, überladen empfunden. Es geht offensichtlich nicht so sehr um die Inkriminierung mangelnder Kreativität, als vielmehr um übertriebenen Gebrauch bzw. eine Anhäufung metaphorischer Ausdrücke in der gleichen Gesprächssequenz, deren Menge bei einer im Grunde seriösen Angelegenheit unangebracht, oberflächlich, empathielos, ja fast lächerlich wirken kann. Dies mag im folgenden Ratschlag der Talkmasterin Arabella an ein Paar mit großen Eheproblemen der Fall sein: ${ }^{15}$

(1) momo ich muss dir eigentlich sagen, jasmina hat ein sehr großes herz, und sie liebt dich wirklich über alle maßen; und ich glaub irgendwa:nnwenn du über eine bestimmte grenze gehen würdest, dann würde auch ihr (.) die geduldsschnur reißen und zwar berechtigterweise; nichtsdestotrotz solltest du bedenken, (.) was du an ihr hast; was du an deiner familie hast; (.) und äh das nicht zu leicht aufs spiel setzen mit dem flirten mit irgendwelchen- (.) zwar sehr hübschen und sehr charmanten Damen, aber doch nur mit irgendwelchen bekanntschaften die das nie aufwiegen können, was du in deine:r . ehe auch hast und in deiner Familie; ja, aber das ist etwas dass ihr miteinander besprechen sollt, das solltest du wirklich mal mit jasmina ehrlich auch drüber sprechen, ihr zwei, unter vier Augen; ja,

Was in diesem Fall zur Häufung idiomatischer Ausdrücke hinzukommt, ist offensichtlich die vermehrte Verwendung recht banaler ${ }^{16}$ Metaphern zur Beschreibung von Liebe und Beziehung, die „etwas Besseres verdienen würden“.

In den Medien wird insbesondere auch die Vermischung von Metaphern, deren Bilder nicht zusammenpassen, angeprangert: „Auch als Hahn im Korb sollten Sie sich nicht vor den Karren spannen lassen“ oder „Mit unserem Zugpferd reiten Sie garantiert auf der Erfolgswelle“. ${ }^{17}$

Auch wenn mangelnde Kreativität prinzipiell auf „einfache“ Wiederholung des bereits Existierenden zurückgeführt werden kann, ermöglichen die angeführ-

15 Die entsprechenden Idiome sind unterstrichen. Die Sequenz stammt aus der Talkshow Arabella des Senders Pro Sieben vom 30.6.1998.

16 Natürlich schon wieder eine persönliche Wertung meinerseits.

17 www.annika-lamer.de/sahnestucke-im-werbetext-so-verwenden-sie-metaphern-richtig (Stand: 11.5.2017). 
ten Fälle kritisch betrachteter Phrasemverwendung doch eine differenziertere Sichtweise auf die Problematik. In der Tat scheinen idiomatische Ausdrücke dann abgelehnt zu werden, wenn sie in zu starker Häufung auftreten, wenn sie in stereotyper Weise für die Bezeichnung gleicher Sachverhalte dienen, wenn sie als veraltet gelten, wenn der bezeichnete Sachverhalt zu ernst ist, als dass er mit klischeehaften Ausdrücken benannt werden könnte, wenn die verwendeten Bilder nicht kompatibel sind.

\section{Linguistische Sprachkritik - Vorgeformtes als sprachliche Formulierungsressource}

Angesichts fehlender trennscharfer Kriterien und sprachwissenschaftlicher Methodik und Empirie laienlinguistischer Kritik an Vorgeformtem ist es kaum überraschend, dass „kritische Semantik“ (vgl. Stein 2016: 144) und linguistisch begründete Sprackkritik zu einem wesentlich differenzierteren Urteil bzgl. der Verwendung phraseologischer Ausdrücke gelangen: ${ }^{18}$

Für eine linguistisch begründete Sprachkritik stellt Vorgeformtheit gleich welcher Komplexität per se keinen Stein des Anstoßes dar. Denn aus der formulierungstheoretischen Perspektive liefert die Strategie reproduzierenden Verbalisierens für eine Vielzahl kommunikativer Anforderungen angemessene und akzeptierte Formulierungsresultate. Textproduzenten, die sich bei der Bewältigung kommunikativer Probleme und Aufgaben ganz oder teilweise gegen unikale Lösungswege entscheiden, verfügen nicht nur über einen Vorrat an habituellen und/oder gesellschaftlich etablierten, d.h. konventionellen Formulierungsweisen, sondern sind auch bereit bzw. sehen sich aufgrund der Konventionen u.U. auch „gezwungen“, auf diesen Vorrat zurückzugreifen und sich Routinen zunutze zu machen. (Stein 2016: 146) $)^{19}$

Gülich (2008: 877) betrachtet dementsprechend den Rückgriff auf Vorgeformtes ganz im Gegenteil als Formulierungsressource:

[...] le recours au préformé n'est pas simplement une activité de reproduction d'éléments " préfabriqués » que le locuteur peut préférer ou non à une production libre ou créative

18 Stumpf (2016: 98) stellt allerdings fest: „Bei genauerer Betrachtung der bisherigen Phraseologie- und Sprachkritikforschung zeigt sich, dass es generell nur wenige linguistisch fundierte Auseinandersetzungen mit Phrasemen bzw. formelhaften Wendungen aus sprachkritischer Perspektive gibt.

19 Siehe auch das Editorial von Stumpf (2016). 
(selon un « idiom principle » ou un « open choice principle »), mais il fait partie - tout comme le recours à d'autres procédés de production discursive (p.ex. la reformulation) - du travail de formulation et est soumis à un traitement interactif.

Es wird dabei keineswegs bestritten, dass etwas bereits in einer bestimmten mehr oder weniger festen Form Existierendes wiederverwendet wird. Vergessen werden darf aber nicht, dass der Sprecher erst einmal ein sprechsituationsadäquates Phrasem auswählen und dieses in den entsprechenden Ko- und Kontext einpassen muss, wobei er häufig unterschiedlichste Transformationen und Veränderungen vorzunehmen hat; sich überdies auf die vermuteten Kenntnisse, Erwartungen und vollzogenen kommunikativen Aktivitäten des Kommunikationspartners einstellen muss. Genau die gleiche Feststellung trifft im Übrigen auch auf alle anderen Entscheidungen des Sprachproduzenten zu. Der einzige Unterschied besteht darin, dass die lexikalische „Vorbelastung“ bei Idiomen größer ist als bei anderen Konstruktionstypen. ${ }^{20}$

\subsection{Zur konversationellen Bearbeitung von Idiomen}

Die Formulierungsarbeit von Phrasemverwendern behandelt Schmale in seinen Arbeiten zur konversationellen Bearbeitung idiomatischer Ausdrücke (z.B. Schmale 2013). ${ }^{21}$ Schon allein die Tatsache, dass die verwendete Idiomform mehrheitlich nicht der (einschlägiger) Phrasem-Wörterbücher entspricht, beweist, dass Sprecher nicht stupide existierende Formeln nachbeten. So modifiziert ein 16-jähriger Talkshow-Gast das bekannte Sprichwort „Früh übt sich, was ein Meister werden will“ zu „Früh übt sich, wer ein Meister sein will“, wobei er eine modernisierte - und im Grunde grammatisch korrektere - Form des Relativpronomens (wer anstelle von was) verwendet und auch ein anderes Kopulaverb gebraucht. Ein anderer Gast verwendet das Idiom „das wurde als bare Münze genommen“, wobei er ebenfalls die in dieser Form veraltete, nicht mehr produktive Form der Präposition (als anstelle von für) ersetzt. Und ein dritter Talkgast sagt selbstkritisch: „ich sitz selbst im Glashaus ich darf nicht mit Steinen werfen“, passt dabei das Sprichwort „Wer im Glashaus sitzt, sollte nicht mit Steinen werfen“ perfekt seiner persönlichen Situation an. Die vorstehenden drei Sequenzen sollen genügen, um aufzuzeigen, in welchem Maße Sprecher bei der Idiomver-

20 Siehe auch die Monographie von Stumpf (2015).

21 Siehe die Literaturliste zu den zahlreichen Arbeiten in unserem Editorial der Nummer 62 von Linguistik online (siehe Schmale 2013). 
wendung kreativ werden. Der Formulierungserfolg kann dabei sogar bis zur Neuschöpfung einer phrasemähnlichen Konstruktion führen.

(2) Talkmaster Meiser befragt Talkgast Jacqueline über ihren Partner Michael. (HM/3-6/13) 22

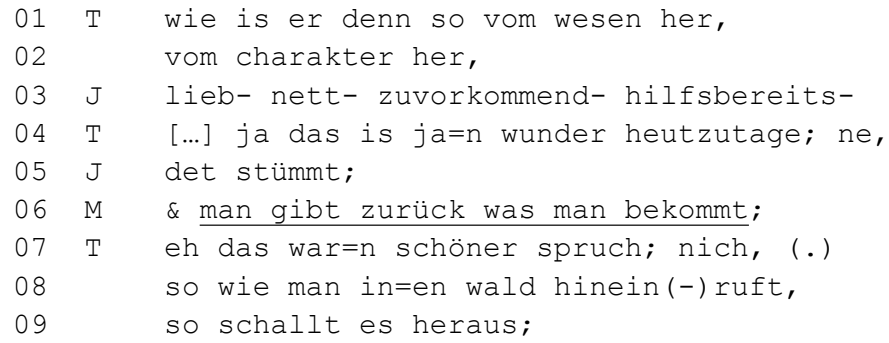

Wenn der Talkmaster Meiser die von $\mathrm{M}$ in Zeile 06 produzierte Konstruktion als „schönen Spruch“ qualifiziert, ${ }^{23}$ dann vielleicht wegen des Vergleichs, vor allem aber wohl wegen des jambisch-anspringenden Rhythmus der Wendung.

So wie in der vorstehenden Sequenz der Talkmaster eine Sequenz heteroparaphrasiert, so gibt es in den untersuchten Talkshows eine Reihe unterschiedlicher Typen konversationeller Bearbeitung phraseologischer Ausdrücke, insbesondere von Idiomen. Hier sollen exemplarisch drei Typen konversationeller Bearbeitung skizziert werden, die in besonders deutlicher Weise die kreative Aktivität des Idiomproduzenten belegen: Auto-Paraphrasen von Idiomen oder durch Idiome; metakommunikative Kommentare von Idiomen sowie Wortspiele mit Idiomen, drei Typen, die die kreative Leistung von Sprachproduzenten besonders anschaulich machen.

- Auto-Paraphrasen von Idiomen oder durch Idiome zeigen sehr deutlich die kreative Kapazität von Sprechern. Dann, wenn Talkgast Jacky ihre eigene, nicht idiomatische Konstruktion durch ein Idiom auto-paraphrasiert (sie spricht über ihren Mann, der sie verlassen will): „aber ich kann ihn ja auch nich festhalten, ich kann ihn ja nich in Ketten legen“ (sic!). Oder auch, wenn Patrizia auf die Frage des Talkmasters nach den Gründen für ihr Übergewicht antwortet: , aber auch n großer Grund is eigentlich dass ich sehr gerne esse auch Essen zubereite- Gäste einlade- .s und kein Kostverächter bin also“. Andererseits werden auch Idiome

22 Aus der Talkshow Hans Meiser des Senders RTL vom 3.6.1998.

23 Und sie dann, allerdings falsch, da die logische Relation umkehrt, durch ein Sprichwort paraphrasiert. 
durch nicht-phraseologische Ausdrücke auto- oder heterophrasiert, wenn eine Krankenschwester zu einem Patienten sagt: „man kann nich alle über einen kamm scheren, man muss eigentlich jeden individuell betreuen“. Oder wenn man im Fußballstammtisch Doppelpass hört: „fast das zeitliche gesegnet hätte fc maskottchen hennes, in köln hieß es schon- ((betonend)) der geißbock ist tot“, so beweist dies, dass Kommunikationsbeteiligte keineswegs einfach Existierendes nachbeten, sondern Idiome kreativ in Ko- und Kontexte einbetten und diese sogar paraphrasieren oder zur Paraphrase einsetzen.

- Metakommunikative Kommentare von Idiomen. Die Tatsache, dass sich Sprecher überdies der Tatsache bewusst sind, dass sie Vorgefertigtes verwenden, wird durch die Metakommentierung von Idiomen mehr als deutlich. Hier einige Beispiele: „ich denke einfach mal- kennt ihr nich den spruch leben und leben lassen; sie leben damit wie die made im speck sacht so mancher; was sich liebt das neckt sich oder wie war das“. Diese vor- oder nachgestellten Kommentare zeigen, dass Sprecher Idiome sehr gezielt einsetzen, auch wenn sie sich bewusst sind - oder vielleicht gerade, weil sie sich bewusst sind -, dass sie einen vorgefertigten Ausdruck verwenden. Aber einen Ausdruck, der ,allgemein“ bekannt ist und deshalb argumentativen Wert besitzen könnte.

- Wortspiele mit Idiomen. Ganz besonders offensichtlich wird die kreative Leistung eines Idiomverwenders dann, wenn Wortspiele damit gemacht werden. Hier Heinz Schenks ${ }^{24}$ Viagra-Witz:

(3) ein mann sitzt in der kneipe, hat einen haufen viagra pillen vor sich liegen und steckt sich laufend welche in die ohren; kommt ein freund dazu und sagt, die musst du schlucken, nicht in die ohren stecken, doch doch das ist schon richtig, mein arzt hat mir gesagt, ich soll die ohren steifhalten;

Oder, weniger anzüglich, aber ebenso klar die semantische Leistung des Sprechers illustrierend: „es gibt tatsächlich so etwas wie:::- medizinische wunder; sie war 1984 total blind- ... vor liebe, inzwischen kann sie wieder sehn (das Talkshow-Publikum lacht)““. ${ }^{25}$ Ebenso wie, über den verlassenen Lebenspartner eines Gastes sprechend: „dann haben sie den in die wüste geschickt... oder im rhein versenkt“. Hier ersetzt Meiser nicht nur das Lexem Wüste, sondern adaptiert das

24 Des legendären Wirtes der ARD-Sendung „Der blaue Bock“ (NDR-Talkshow vom 5.2.1999). 25 Vom Talkmaster Hans Meiser in einer seiner gleichnamigen Shows so geäußert. 
neue, Rhein, auch noch an die Kommunikationssituation, die Talkshow findet nämlich in Köln statt.

\subsection{Verwendung idiomatischer Ausdrücke in der Werbung und in Schlagzeilen}

Sollte es noch eines Beweises bedürfen, dass die Verwendung von Idiomen alles andere als unkreativ ist, hier einige Beispiele aus der Werbung und aus Zeitungsschlagzeilen. ${ }^{26}$ So titelt die Bild-Zeitung vom 23.9.2011: „Papst nimmt Politiker ins Gebet!“, oder die TaZ vom 19.2.2013: „Bundesregierung beschließt Mali-Einsatz. Deutsche in den Sand gesetzt.“ Ganz besonders kreativ ist die französische Wochenzeitung Le Canard Enchaîné, in der systematisch Wortspiele, sehr häufig mit Idiomen, in der Hauptschlagzeile zu finden sind, z.B. „Difficile accord VertsPS entre centrales nucléaires et circonscriptions. Un rude accouchement par les sièges“ (no. 4752 vom 23.11.2011). Reichhaltige semantische Implikationen zeugen von großer Kreativität des Autors der jeweiligen Schlagzeile, der sich jeweils der im Kontext aktualisierbaren wörtlichen Bedeutung des Idioms bedient:

- ins Gebet nehmen: jemanden ermahnen oder zurechtweisen vs. für jemanden beten (es geht um den Papst);

- in den Sand setzen: etwas selbst verschulden, scheitern vs. eine Person oder Sache in den Sand (ab)setzen (hier: die deutschen Soldaten);

- accouchement par le siège: eigentlich 'Steißgeburt', hier verändert in les sièges', da es um die schwierige Verteilung von Wahlkreisen für künftige Abgeordnetenmandate geht.

Der endgültige, wenn noch nötige Beweis der absoluten Kreativität der Idiomverwendung kann über die Verwendung idiomatischer Wendungen in der Werbung erbracht werden: „Rolletarier aller Länder vereinigt euch“ (Werbung für Zigarettenpapier); „Große Klappe, viel dahinter“ (Werbung für VW Polo Variant); „Wir machen Ihnen schöne Beine“ (Reklame für Nylonstrüpfe). Die Originalität besteht gerade darin, Phraseme oder Zitate ${ }^{27} \mathrm{zu}$ verändern, eine wörtliche Bedeutung zu aktualisieren, gleichzeitig aber aufgrund ihres Bekanntheitsgrades die idiomatische Bedeutung zu evozieren. Vorrangig wohl, um Belustigung und darüber ver-

26 Siehe dazu die einschlägige Monographie von Balsliemke (2001).

27 Zitate stellen laut Burger (2010) eine phraseologische Spezialklasse dar. Die Nennformen der veränderten Phraseme lauten: Proletarier aller Länder vereinigt euch; Große Klappe, nichts dahinter; jmdm. Beine machen. 
mittelt Aufmerksamkeit zu erzeugen. Auf keinen Fall jedoch liegt hier die Verwendung abgenutzter oder veralteter Phraseme vor, wäre dies der Fall, würden hochqualifizierte Werbetexter nicht auf diese zurückgreifen.

Abschließend soll diese These über die kurze Analyse zweier ZeitschriftReklamen belegt werden. Die semantischen Beziehungen der Birkel-Reklame mit dem ein nominales Idiom ${ }^{28}$ enthaltenden Slogan „Was man nicht alles für so eine halbe Portion tut" sind äußerst komplex (siehe Abb. 1). Das Foto zeigt zwei Kinder, die sich eine Portion Spaghetti teilen, aktualisiert folglich die wörtliche Bedeutung der Nominalphrase eine halbe Portion. ${ }^{29}$ Doch gleichzeitig, vorausgesetzt, man kennt sie, wird die phraseologische Bedeutung 'ein kleiner Mensch' evoziert, die natürlich auf die beiden Kinder des Fotos Bezug nimmt.

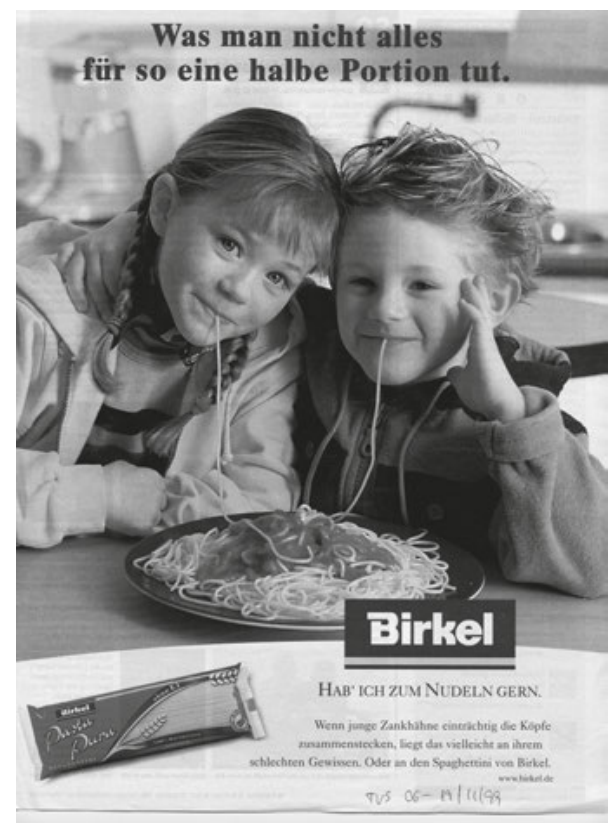

Abb. 1: Reklame für Birkel-Spaghetti aus der TV Spielfilm vom 19.11.1999

28 eine halbe Portion ein nicht ernst zu nehmender kleiner, schwächlicher, unscheinbarer usw. Mensch.

29 Die Konstruktion 'Was man nicht für + NP + tut' lassen wir außen vor. 
Noch komplexer wird es in der folgenden Werbung mit dem Slogan „Viele Menschen haben 1997 ihr Herz verschenkt. 562 davon waren Organspender“ (siehe Abb. 2). Das im Werbeslogan enthaltene Idiom sein Herz verschenken hat hier wörtliche Bedeutung, bezieht sich folglich also auf die Organspende. Da aber eine bildliche Darstellung dieser Tatsache ganz offensichtlich eher makaber wäre, zeigt das Foto ein sich umarmendes Liebespaar, das im übertragenen Sinne sein Herz als Symbol der Liebe verschenkt hat. Diese Werbung stellt im Übrigen einen Ausnahmefall dar, das materielle Bild symbolisiert die phraseologische Bedeutung, in der Regel wird nämlich durch ikonographische Elemente die wörtliche Bedeutung aktualisiert. Die Werbetexter waren also ganz besonders kreativ in diesem Fall.

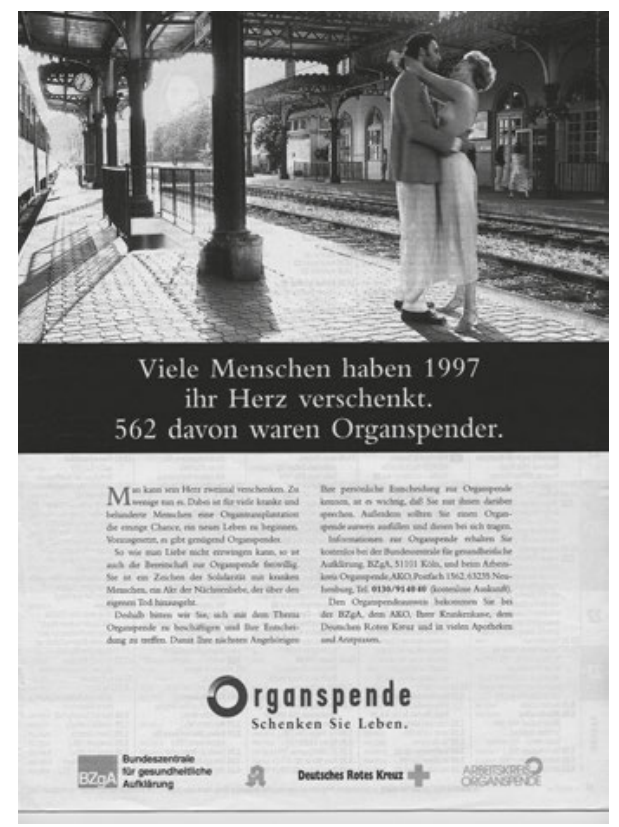

Abb. 2: Reklame für Organspenden im Jahr 1997

\section{Größere Kreativität durch völligen Verzicht auf Idiome?}

Anstelle eines Fazits soll als abschließende Falsifizierung der These von der Unkreativität idiomatischer Ausdrücke die Frage gestellt werden, ob der folgende Text, es handelt sich um einen Ausschnitt aus Thomas Manns Zauberberg, durch 
eine „Entidiomatisierung“ oder „Entphraseologisierung“ kreativer würde. Wenn die These der praktischen Stilistik generell auf „Konserven“, also auch auf Idiome zutrifft, dann müssten ebenfalls die, die Thomas Mann dem Hofrat in den Mund legt, als solche bewertet werden. ${ }^{30}$

(4) „Sie sind eine Art von Feigling und Duckmäuser, Mensch, und wenn Ihr Vetter Sie einen Zivilisten nennt, so ist das noch sehr euphemistisch ausgedrückt.«

»Kann alles sein, Herr Hofrat. Natürlich, die Schwächen meines Charakters stehen doch außer Frage. Aber das ist es eben, daß sie im Augenblick wohl außer Frage stehen, und was ich Sie schon seit drei Tagen bitten wollte, ist nur $-\ll$

»Daß ich Ihnen recht angenehm gezuckerten und gepanschten Wein einschenke! Sie wollen mich behelligen und mich langweilen, damit ich Sie in ihrer verdammten Duckmäuserei befestige und damit Sie in Unschuld schlafen können, während andere Leute wachen und sich den Wind um die Nase wehen lassen."

»Aber, Herr Hofrat, Sie sind recht streng mit mir. Ich wollte im Gegenteil-«

»Ja, Strenge, das ist nun gerade gar nicht Ihre Sache. Da ist Ihr Vetter ein anderer Kerl, von anderem Schrot und Korn. Der weiß Bescheid. Der weiß schweigend Bescheid, verstehen Sie mich? Der hängt sich den Leuten nicht an die Rockschöße, um sich blauen Dunst und Harmlosigkeit vormachen zu lassen. Der wußte, was er tat und was er daransetzte, und ist ein Mannsbild, das sich auf Haltung versteht und aufs Maulhalten, was eine männliche Kunst ist, aber leider nicht die Sache von solchen bipedischen Annehmlichkeiten wie Sie. Aber das sage ich Ihnen, Castorp, wenn Sie hier Szenen aufführen und ein Geschrei erheben und sich Ihren Zivilgefühlen überlassen, so setze ich Sie an die Luft. Denn hier wollen Männer unter sich sein, verstehen Sie mich.«(Thomas Mann, Der Zauberberg, 1924: 558)

Völlig abgesehen davon, dass es sich um einen Dialog Thomas Manns handelt, wer wollte ernsthaft behaupten, hier handele es sich um unkreativen Sprachgebrauch. Dabei werden auch hier, wie in der o.a. Talkshow-Sequenz (siehe (1)), Metaphern wie sich an die Rockschöße hängen oder an die Luft setzen verwendet. Liegt es an der Qualität der Metaphern, dass diese im Gegensatz zu den von Arabella gebrauchten nicht als fade oder inadäquat eingestuft werden? Oder doch

30 Sie wurden in der folgenden Sequenz unterstrichen. 
daran, dass sie von Thomas Mann produziert wurden? Im Grunde gibt es hierauf keine wissenschaftliche, semantisch oder durch Gebrauchshäufigkeit begründbare Antwort. Das wahre Motiv sind ganz einfach Rezipienten-Präferenzen: Dem einen gefällt's, dem anderen nicht! Vielleicht deshalb, weil letzterer ein höher entwickeltes Sprachbewusstsein besitzt, mehr liest, mehr mit Sprache zu tun hat, aber selbst das ist nicht sicher.

Was aber unbestreitbar ist: Nicht jeder Idiomgebrauch verstößt prinzipiell gegen das variatio-delectat-Gebot. Die angeführten Beispiele haben belegt, dass idiomatische Ausdrücke als kreative Formulierungsressource zum Einsatz kommen.

\section{Literatur}

Altenberg, Bengt (1998): On the phraseology of spoken English: the evidence of recurrent word combinations. In: Cowie, Anthony Paul (Hg.) (1998): Phraseology. Theory, analysis and applications. Oxford: OUP. 101-122.

Auer, Peter (2016): „Wie geil ist das denn?“ Eine neue Konstruktion im Netzwerk ihrer Nachbarn. In: Zeitschrift für Germanistische Linguistik 44, 1. 69-92.

Bally, Charles (1909): Traité de stylistique française. Paris: Klincksieck.

Balsliemke, Petra (2001): ,Da sieht die Welt schon anders aus.' Phraseologismen in der Anzeigenwerbung. Modifikation und Funktion in Text-Bild- Beziehungen. Baltmannsweiler: Schneider.

Bardosi, Vilmos/Ettinger, Stefan/Stölting, Cécile (2003): Redewendungen Französisch/ Deutsch. Thematisches Wörter- und Übungsbuch. 3. Aufl. Tübingen/Basel: Francke.

Bréal, Michel (1872): Quelques mots sur l'instruction publique en France. Paris: Hachette. Burger, Harald (1989): Bildhaft, übertragen, metaphorisch - Zur Konfusion um die semantischen Merkmale von Phraseologismen. In: Gréciano, Gertrud (Hg.): Europhras 1988. Phraséologie contrastive: Actes du Colloque International, Klingenthal - Strasbourg 12-16 mai 1988. (= Collection Recherches Germaniques 2). Strasbourg: Université des Sciences Humaines, Département d'Études d'Allemand. 17-29.

Burger, Harald (2010): Phraseologie - Eine Einführung am Beispiel des Deutschen. 4., neu bearb. Aufl. (= Grundlagen der Germanistik 36). Berlin: Schmidt.

Camilleri, Andrea (2003): L'odeur de la nuit. Paris: Fleuve Noir. [Deutsch: Der Kavalier der späten Stunde. Commissario Montalbanos 6. Fall. Köln: Bastei Lübbe.].

Drescher, Martina (1997): Wie expressiv sind Phraseologismen? In: Sabban, Annette (Hg.): Phraseme im Text: Beiträge aus romanistischer Sicht. (= Studien zur Phraseologie und Parömiologie 14). Bochum: Brockmeyer. 67-95.

Erman, Britt/Warren, Beatrice (2000): The Idiom Principle and the Open Choice Principle. In: Text 20, 1. 29-62.

Gréciano, Gertrud (1983): Signification et dénotation en allemand: La sémantique des expressions idiomatiques. (= Recherches Linguistiques IX). Paris/Metz: Klinksieck.

Gülich, Elisabeth (2008): Le recours au préformé: une ressource dans l'interaction conversationnelle. In: Durand, Jacques et al. (Hgg.): Congrès mondial de linguistique française. Paris, 9-12 juillet 2008. Paris: ILF/EDP Sciences. 
Lakoff, George/Johnson, Mark (1998): Leben in Metaphern: Konstruktion und Gebrauch von Sprachbildern. Heidelberg: Auer.

Magri-Mourgues, Véronique/Rabatel, Alain (2015a): Répétitions et genres. Le discours et la langue 72. Paris: L'Harmattan.

Magri-Mourgues, Véronique/Rabatel, Alain (2015b): Pragmatique de la répétition. Semen 38. Besançon: PUFC.

Mann, Thomas (1982): Der Zauberberg. Frankfurt a.M.: Fischer.

Monneret, Philippe (2010): Expressivité et image: Retour sur la conception Guillaumienne de l'expressivité. In: Gautier, Laurent/Monneret, Philippe (Hgg.): La fonction expressive. Bd. 2. Besançon: PUFC. 19-38.

Paul, Hermann (1970 [1880]): Prinzipien der Sprachgeschichte. 8. Aufl. Tübingen: Niemeyer. Riesel, Elise (1970): Der Stil der deutschen Alltagsrede. Leipzig: Reclam.

Schmale, Günter (2008): Conceptions populaires de la conversation. In: Achard-Bayle, Guy/ Paveau, Marie-Anne (Hgg.): Linguistique Populaire? Pratiques 12. 58-80

Schmale, Günter (2010): Ist ein idiomatischer Ausdruck immer expressiv? Korpusbasierte und fragebogengestützte Beobachtungen zu einer verbreiteten Prämisse. In: Yearbook of Phraseology I. Berlin/New York: De Gruyter. 97-124.

Schmale, Günter (2013): Forms and functions of idiomatic expressions in conversational interaction. In: Schmale, Günter (Hg.): Formen und Funktionen vorgeformter Konstruktionseinheiten in authentischen Konversationen/Forms and functions of formulaic construction units in conversation. Linguistik online 62. 67-96. (www.linguistik-online. de/62_13/schmale.html, Stand: 11.5.2017).

Stein, Stephan (1995): Formelhafte Sprache. Untersuchungen zu ihren pragmatischen und kognitiven Funktionen im gegenwärtigen Deutsch. Frankfurt a.M. u.a.: Lang.

Stein, Stephan (2016): Vorgeformtheit im öffentlichen Sprachgebrauch zwischen laienlinguistischer und linguistisch begründeter Sprachkritik: „Phrasen“, „Floskeln“ und „Schablonen“vs. Formulierungsmuster, -stereotype und -routinen. In: Aptum. Zeitschrift für Sprachkritik und Sprachkultur. Themenheft: Formelhafte Sprache und Sprachkritik 12/2.133-151.

Stumpf, Sören (2015): Formelhafte (Ir-)Regularitäten. Korpuslinguistische Befunde und sprachtheoretische Überlegungen. (= Sprache - System und Tätigkeit 67). Frankfurt a.M.: Lang.

Stumpf, Sören (2016). Editorial: Formelhafte Sprache und Sprachkritik. In: Aptum. Zeitschrift für Sprachkritik und Sprachkultur 12, 2. 97-102.

Van Lancker-Sidtis, Diana/Rallon, Gail (2004): Tracking the incidence of formulaic expressions in everyday speech: methods for classification and verification. In: Language \& Communication 24, 3. 207-240.

Wray, Alison (2000): Formulaic sequences in second language teaching: Principle and practice. In: Applied Linguistics 21, 4. 463-489.

Wray, Alison/Perkins, Michael R. (2000): The functions of formulaic language. An integrated model. In: Language \& Communication 20.1-28. 